\title{
Of sets of offsets: Cumulative impacts and strategies for compensatory restoration
}

\author{
Thébaud Olivier ${ }^{1,{ }^{*}}$, Boschetti Fabio ${ }^{2,3}$, Jennings Sarah ${ }^{4}$, Smith A. Graham ${ }^{5}$, Pascoe Sean ${ }^{1,5}$
}

${ }^{1}$ CSIRO Oceans and Atmosphere Flagship, Brisbane, Australia

2 Ifremer, UMR M101, AMURE, Unité d'Économie Maritime, Brest, France

${ }^{3}$ University of Western Australia, Perth, Australia

${ }^{4}$ University of Tasmania, Hobart, Australia

${ }^{5}$ CSIRO Oceans and Atmosphere Flagship, Hobart, Australia

* Corresponding author, Olivier Thébaud, Tel.: +33 29822 4989; fax: +33 298224776 ;

email address : olivier.thebaud@ifremer.fr

\begin{abstract}
:
Biodiversity offsets are increasingly advocated as a flexible approach to managing the ecological costs of economic development. Arguably, however, this remains an area where policy-making has run ahead of science. A growing number of studies identify limitations of offsets in achieving ecologically sustainable outcomes, pointing to ethical and implementation issues that may undermine their effectiveness. We develop a novel system dynamic modelling framework to analyze the no net loss objective of development and biodiversity offsets. The modelling framework considers a marine-based example, where resource abundance depends on a habitat that is affected by a sequence of development projects, and biodiversity offsets are understood as habitat restoration actions. The model is used to explore the implications of four alternative offset management strategies for a regulator, which differ in how net loss is measured, and whether and how the cumulative impacts of development are considered. Our results confirm that, when it comes to offsets as a conservation tool, the devil lies in the details. Approaches to determining the magnitude of offsets required, as well as their timing and allocation among multiple developers, can result in potentially complex and undesired sets of economic incentives, with direct impacts on the ability to meet the overall objective of ecologically sustainable development. The approach and insights are of direct interest to conservation policy design in a broad range of marine and coastal contexts.
\end{abstract}

\section{Highlights}

- We develop a novel system dynamics modelling framework to address biodiversity offsets. $\longrightarrow$ This framework is applied to a marine habitat-resource system. We use this to examine the effectiveness of alternative offset management strategies. Simulations show that some strategies can result in undesired economic incentives.

Keywords: Biodiversity offsets, Compensatory restoration, Cumulative impacts, Habitat-resource interactions, Bio-economic modelling, Social acceptability 


\section{Introduction}

Biodiversity offsets are increasingly considered as an option to compensate for the ecological costs of development, with 72 countries identified as having some form of legislative requirement for compensatory biodiversity restoration either already in place or under development (Madsen et al. 2011). The growing popularity of voluntary offsets is also noted, with the use of offsets expected to increase in the future (Maron et al. 2012). Biodiversity offsets refer to actions taken at an offset site intended to compensate for a loss of biodiversity at an impact site. They may include a wide range of interventions, at species, community or whole-of-ecosystem levels, which can be carried out as part of voluntary or mandatory regimes, with the aim to compensate for on-going and anticipated ecological loss. While often vaguely defined, the objective of no net loss" (Gordon et al. 2011, Gardner et al. 2013) is central to offsets and increasingly offset policies require demonstration of the equivalence between what is created and what is lost.

Despite their increasing popularity as a flexible approach to the reconciliation of economic development with biodiversity conservation, a number of studies have pointed to the potential limitations of offsets in achieving ecologically sustainable outcomes (Morris et al. 2006, Maron et al. 2012). In particular, studies have demonstrated that the no net loss objective may only be achieved with high offsets ratios (i.e. where more ecological assets are protected or created than are lost) if at all, and with intensive monitoring efforts, even in cases that would normally be considered ideal for implementation of an offset strategy based on biology and habitat characteristics (Pickett et al. 2013). Where such characteristics are not present, there is a risk that the offsets may not provide services equivalent to those that were lost. In such cases, the development of markets in which offsets are traded may in fact increase, rather than decrease, environmental degradation, as Palmer and Filoso (2009) discuss in the context of aquatic ecosystems.

In a recently published review of offset-focused biodiversity restoration programs, Maron et al. (2012) identify a number of potential limitations to offsets, which include time lags in the recovery of ecological systems. Indeed, understanding of key ecological, as well as social responses, will be crucial in determining circumstances under which offsets may be (in) effective as biodiversity conservation tools. First, ecological responses to the implementation of an offset, and in particular delays and threshold effects, will determine when, if ever, a set of impacted services is recovered. Second, societal responses to perceived (vs. actual) changes in the availability of ecological services may influence acceptability of an offset action. Third, although difficult to predict (Mitchell and Parkins 2011), lags in societal response to losses of ecosystem services may in turn affect the potential effectiveness of offsets. These lags may arise from a lack of understanding of ecological systems, poor measurement of system properties or natural inertia in the social processes that determine collective action.

Here, we explore several conceptual issues relating to the effectiveness of alternative biodiversity offsetting approaches, using a marine system as an example. Where offset policies apply equally to coastal and marine systems as to terrestrial, as is the case for Australia's Commonwealth Government's offset policy (Department of Sustainability 2012), experience with their use in the marine context is generally less well developed. We propose a modeling framework to assess stylized offset management strategies under alternative scenarios relating to (i) ecological response to the implementation of multiple developments and offset actions - or sets of offsets, in particular the time delays involved in ecological recovery, and (ii) societal response to the damages caused by development, which determines the objectives for compensatory restoration actions. In particular, we consider circumstances in which actual losses of ecosystem services do not directly translate into a policy requirement for restoration. This may be due, first, to a lack of awareness of the loss 
of service, because actual losses are difficult to detect, at least until they become relatively large. In addition, even if losses are detectable, it may still be difficult to evaluate their full extent (due for example to lack of historical knowledge of the ecosystem or to lack of previous analogous changes). Second, people may be willing to accept low levels of losses in ecosystem services, such that actual losses, although detected, are not perceived as being problematic, or that the expected benefits of imposing constraints on development are not seen to outweigh the costs of doing so, including in particular the coordination costs of collective action.

Our management strategies capture differences in the way in which the no net loss" objective is interpreted, including restoration scaling approaches that rely on habitat-tohabitat modeling, and value-to-value methods that explicitly account for the value to society of ecosystem changes. While the former approaches are the most commonly encountered interpretations of the no net loss" objective, it can be argued that offsets should be determined in relation to the value of lost ecosystem services, such that the objectives of a biodiversity offset policy should be defined in terms of no net value loss".

The management strategies considered in this analysis also capture contexts in which developments are assessed and approved, and offsets determined, on a project-by-project basis despite being components of a regionally-based strategic approach. Consistent with the conservation biology literature on this topic, the analysis focuses on the case of so-called -direct offsets" involving the provision of either averted ecological losses or restoration gains at least equivalent to the ecological impacts of a particular development in a given region (see model description below), rather than offsets involving actions with indirect outcomes such as financial compensation (Maron et al. 2012).

The modeling framework we propose is built to reflect a marine-based example, where the abundance and hence utility of a biological resource depends on a habitat that is affected by development or exploitation. This is representative of many marine situations involving fish and shellfish species of commercial interest, and their dependence on, for example, seagrass (Anderson 1989), mangrove (Barbier et al. 2002) and coastal marshes (Lynne et al. 1981), coral reefs (Foley et al. 2010), freshwater bodies (Knowler et al. 2003) or seafloor habitat (Lindholm et al. 2001). The model captures four main processes spanning both the physical and human components of the system within which offsetting occurs: (i) a biological resource which provides a range of ecosystem services, (ii) a habitat which supports the biological resource and is negatively impacted by economic development, (iii) a regulator which assesses the level of restoration required for a development proposal to be approved and (iv) a social process which determines the permitted extent of ecosystem service loss over a given time horizon.

The model is stylized in that:

- it employs simple logistic equations to describe the dynamics of a single, homogeneous, non-spatially resolved biological resource and its habitat;

- simple equations are used to describe the generation of utility associated with the ecosystem services supported by the resource;

- the level of offsets is limited only by the areal extent of the habitat;

- it is assumed that all important quantities can be accurately and objectively measured; and

- there is no uncertainty in the development impacts and ecological responses to offsets (although the occurrence of lags in detecting and acting upon losses of ecosystem service and in implementing offsets is explicitly represented). 
Although stylized, the model allows exploration of key issues which may arise from the cumulative impacts of approved developments under alternative offset management strategies.

\section{The model}

A conceptual diagram of the model is presented below, and a full description of the model is provided in the appendix. We consider a biological resource, the biomass $X$ of which is a function $F$ of habitat $H$ availability. This biological resource provides a number of ecosystem services, which may include provisioning services (e.g. commercial or subsistence fishing, extraction of molecules with medicinal properties), cultural services (e.g. recreational fishing or diving, aesthetic services), as well as supporting and regulating services (Millennium Ecosystem Assessment 2005). We consider the case of an extractive use, such as commercial fishing with a fixed level of harvesting effort yielding harvest $h$ a function of harvestable biomass. The resource biomass thus evolves according to:

$$
X_{t+1}=X_{t}+F\left(X_{t}, H_{t}\right)-h\left(X_{t}\right)
$$

where $t$ is time. The harvest generated by the fishery produces social welfare which we define as the utility $U_{t}$ derived from this provisioning service. We assume that this can be measured in monetary terms. Given that harvest is fixed, $U_{t}$ is directly proportional to the resource biomass $X_{t}$.

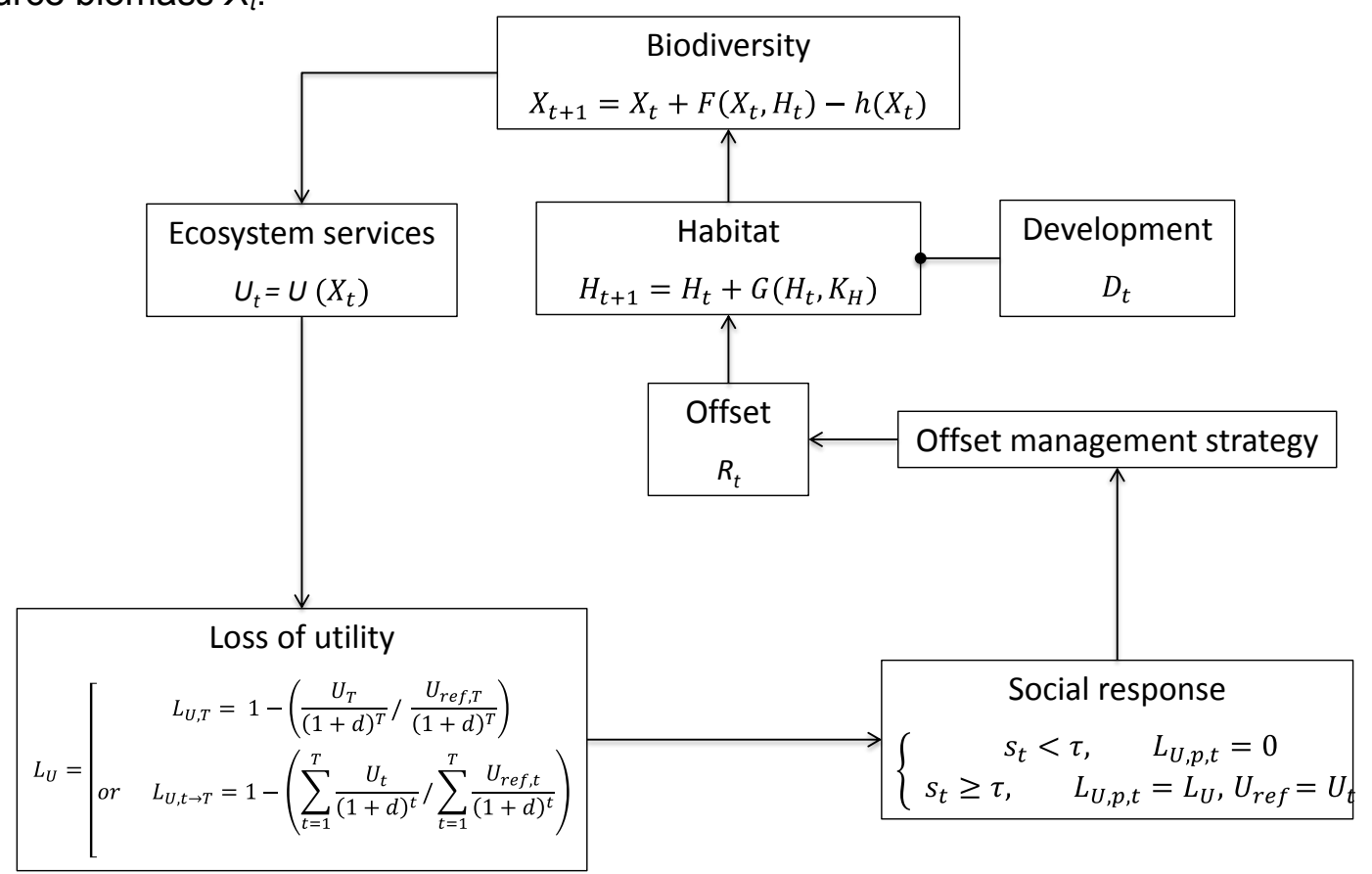

Figure 1. Conceptual diagram of the model. See text for notations and definitions.

We distinguish between disturbances and recovery processes of the habitat, and disturbances and recovery processes of the area available for habitat, which we refer to as the habitat's carrying capacity hereafter. Disturbances to the habitat can occur naturally (e.g. through storm damage), and hence we model habitat recovery, assuming a logistic growth function $G$, as:

$$
H_{t+1}=H_{t}+G\left(H_{t}, K_{H}\right)
$$


where $K_{H}$ is the habitat's carrying capacity. In contrast, disturbances to this carrying capacity occur as a result of development projects (which also remove a corresponding fraction of the habitat). We define $D_{t}$ as the amount of habitat and habitat carrying capacity that is disturbed (removed) by each project at the beginning of a given time step:

$$
\widetilde{H_{t}}=H_{t}-D_{t} ; \widetilde{K}_{H}=K_{H}-D_{t}
$$

Where $\widetilde{H_{t}}$ is the effective amount of post-development habitat and $\widetilde{K}_{H}$ is the revised habitat carrying capacity. A biodiversity offset policy defines the obligation for developers to implement such compensating restoration, or offset, $R_{t}$ which increases the habitat carrying capacity. We assume that only one offset action is undertaken for each development project (the subscript $t$ refers to the time at which the offset action is to be undertaken). For simplicity, we also assume that restoration of $K_{H}$ occurs adjacent to the area disturbed by the development project, such that the offset action provides services to the same communities (both biological and human) affected by the development project. The offset $R_{t}$ is implemented in full in the time step that follows the corresponding development (and hence disturbance) such that:

$$
\widehat{K}_{H, t+1}=\widetilde{K}_{H, t}+R_{t}
$$

Where $\widehat{K}_{H}$ is the revised carrying capacity. Recovery of the habitat following a developmentinduced disturbance will then occur according to equation (2), eventually leading to recovery of the resource (equation 1), of the associated services (in this case commercial harvest of the biological resource) and of the derived utility. We further assume that any restoration costs that could initially be anticipated by the developer have been budgeted as a component of the project itself, and do not therefore affect the decisions of developers regarding development.

\section{Setting objectives for offset policy}

We consider three alternative interpretations of the no net loss" objective, the first reflecting the habitat equivalency criteria which, in our model, requires the level of restoration, $R_{t}$, be determined in direct relation to the level of damage, $D_{t}$. We also consider two value equivalency approaches to no net loss", both of which calculate the required compensating restoration by comparing the difference between the utility that would result from the status quo involving no development, $U_{\text {ref, }}$, and the utility that would result if the development was approved, $U_{t}$, assuming that utility can be measured adequately and is accepted as a single criterion by which to assess gains and losses of ecosystem services. Two alternative definitions of this no net (utility) loss are then considered. In the first, $L_{U, T}$ (Equation 5, top), measures the difference between $U_{T}$ (the level of utility achieved in the last time period $T$ of the planning horizon when development occurs) and $U_{\text {ref. }}$ In the second definition of no net (utility) loss, losses $L_{U, t \rightarrow T}$ (Equation 5, bottom) are evaluated and integrated over the entire planning horizon, thereby reflecting the time discounted cumulative utility loss. This follows (Overton et al. 2013) who suggest that discounting biodiversity values over time provides a more appropriate measure for guiding offset policy making than taking an end point (assumed equal to the initial point in ecological terms) only. We thus have:

$$
L_{U}=\left[\begin{array}{rl}
L_{U, T} & =1-\left(\frac{U_{T}}{(1+d)^{T}} / \frac{U_{r e f, T}}{(1+d)^{T}}\right) \\
\text { or } \quad L_{U, t \rightarrow T} & =1-\left(\sum_{t=1}^{T} \frac{U_{t}}{(1+d)^{t}} / \sum_{t=1}^{T} \frac{U_{r e f, t}}{(1+d)^{t}}\right)
\end{array}\right.
$$

where $d$ is a non-negative discount rate reflecting societal time preference. 
We further allow for the possibility that there may be a lag in society's demand for policymakers to implement an offset policy by explicitly distinguishing between actual and perceived utility loss, $L_{U, p}$ defined as:

$$
\left\{\begin{array}{c}
s_{t}<\tau, L_{U, p, t}=0 \\
s_{t} \geq \tau, L_{U, p, t}=L_{U}, U_{r e f}=U_{t}
\end{array}\right.
$$

where $s$ is a social concern factor assumed to vary as a function of actual utility loss as described below (equation 6), and the parameter $\tau$ is a threshold level above which utility loss is perceived and offsets are required. A defining component of utility loss in equation 5 is the reference (or baseline) level of utility against which it is measured. We assume that this reference level of utility is set at the level of utility existing in the period in which the threshold for action, $\tau$, is reached (see online supplementary information for details and an example of the social response function), but note that the reference utility level could be set by the regulator as that occurring in any particular year.

\section{Simulations}

We use a parameterized version of our model to simulate the outcomes of implementing a schedule of five development projects occurring over consecutive years (years 1 to 5 , which seems to be a common time frame for the implementation of an environmental policy in most OECD countries) under four alternative offset management strategies. Each development project results in the loss of $5 \%$ of the normalized carrying capacity (as well as the corresponding amount of habitat - equation 3) and required offsets are implemented in the period immediately following the development (years 2 to 6 ). Our simulations are run for a 25 -year time period following the first development project $(T=25)$. Our simulations are not intended to represent any particular real world marine system and parameter values and initial conditions of the system (shown in the supplementary materials) are chosen to ensure that the system is in a steady state prior to the first development (year 0), and such that utility derived from the harvest of the biological resource is positive.

We conduct a reference or base case simulation for which we assume an initially severely degraded environment by setting the carrying capacity of the habitat to 0.5 . We also assume a social threshold for action of $\tau=0$, reflecting the case in which society will not tolerate any further net loss of habitat and associated ecosystem services, and a discount rate of $d=0$. Sensitivity of our results to alternative assumptions regarding these key parameters is examined through the analysis of alternative scenarios.

\subsection{Offset management strategies}

We consider four alternative management strategies for implementing offsets, each of which comprises a i) development assessment process and ii) an offset policy objective. We consider two assessment processes (project-by-project and strategic) and the three approaches of no net loss defined in section 3 yielding:

- Strategy 1 - Sequential Myopic: Development assessment is on a project-by-project basis and compensatory restoration is set on a habitat equivalency basis. This implies that the cumulative impacts of development projects that have already occurred, or of their associated offsets, are not accounted for in determining the level of restoration required. The offset schedule is such that:

$$
R_{t}=D_{t}, \quad t=1 \rightarrow 5
$$


- Strategy 2 - Sequential Correcting: Development assessment is on a project-byproject basis (as in Strategy 1) but compensatory restoration for each project is determined on a value equivalency basis, as defined in equations 5 and 6 , considering only losses in the final year $T$. The offset schedule is:

$$
\text { choose } R_{t} \text { s.t. } L_{U, T, t}=0 \quad t=1 \rightarrow 5
$$

- Strategy 3 - Sequential Compensating: as Strategy 2, but accounting for the time discounted cumulative utility loss over the entire planning horizon (equations 5 and $6)$ :

$$
\text { choose } R_{t} \text { s.t. } \quad L_{U, t \rightarrow T, t}=0 \quad t=1 \rightarrow 5
$$

- Strategy 4 - Strategic Assessment: as Strategy 3, but the evaluation of the required level of offsets is made across the entire schedule of development projects and is applied from the initial time period of the simulation with the aim to spread offset obligations uniformly across projects:

$$
\text { choose } R_{t} \text { s.t. } L_{U, 1 \rightarrow T}=0
$$

\section{Results}

\subsection{Reference run}

The results obtained for our reference run are presented in Figure 2. Under the Sequential Myopic strategy (black line) the habitat carrying capacity has been fully restored (Figure 2a, black thick line) by the end of the simulation, but the intrinsic ability of the habitat to recover $\left(r_{H}\right)$ following a disturbance is not strong enough to allow the habitat (or associated ecosystem services) to be fully restored within the 20 year evaluation period (Figure 2a, black dashed line). As a consequence, neither the resource nor utility are able to fully recover to their pre-development levels (Figure $2 \mathrm{~b}$ black dashed line and $2 \mathrm{c}$ black line), resulting in a positive, albeit small, utility loss (Figure $2 \mathrm{~d}$ ).

(a)

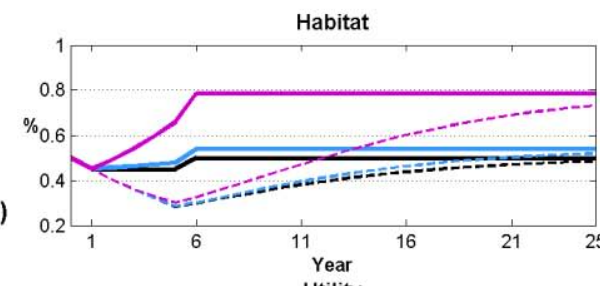

(c)
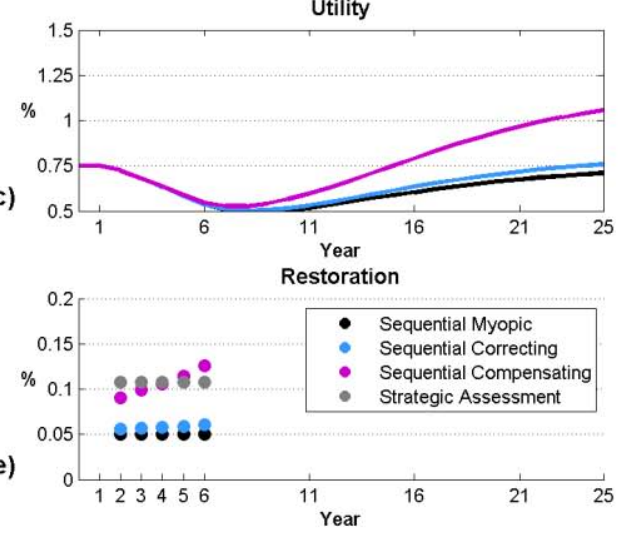

(b)
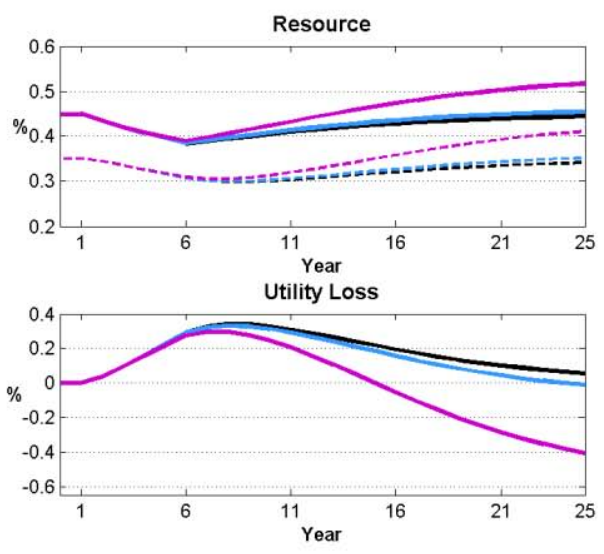
Figure 2. Reference simulation results. Black denotes the Sequential Myopic, cyan the Sequential Correcting, magenta the Sequential Compensating and grey the Strategic Assessment strategy. Panels (a) and (b): habitat and biological resource dynamics for the first three offset management strategies (thick lines represent the evolution of the carrying capacity I habitat carrying capacity (normalized to 1); dashed lines show the evolution of abundances). Panels (c) and (d): evolution of utility and utility loss, respectively, for these three strategies. Panel (e): magnitude and timing of restoration interventions required in the four offset implementation strategies.

The Sequential Correcting strategy (cyan line), requires that developers invest in slightly larger offsets, as compared to the Sequential Myopic strategy (Figure 2e, cyan vs. black dots). This results in an increase in the carrying capacity of the habitat above its predevelopment state which, given the lag in habitat recovery, is required to return the resource abundance, and hence utility, to its baseline level by the end of the simulation period. Indeed, most current offset policies require greater restoration than the expected damage, while in many cases this is justified by inherent risks in terms of restoration (Moilanen et al. 2009). Even in our deterministic model it is however possible that an offset system may generate a higher area of habitat (through, for example, regeneration of other already degraded areas) than was initially damaged by the development.

The Sequential Compensating strategy (magenta line), which fully accounts for cumulative losses arising from lags, requires restoration of the habitat carrying capacity to a level substantially above its pre-development level. Because of the initial decline in utility and the delay in recovering the ecosystem service, compensation for losses incurred during the recovery phase can only be achieved by requiring offsets that considerably increase the resource abundance (and thus utility) at some stage during the recovery period. The magenta line in Figure 2c shows that, given the assumptions of our reference run, there is a net utility gain by the end of the simulation period.

The Sequential Compensating strategy requires considerably larger, and increasing, offsets, than either the Myopic or Correcting strategies (Figure 2e). Clearly, in this case, the timing of the development in the simulation period has a considerable impact on the distribution of offsets required across individual projects. This could potentially lead to difficulties in implementing offsets, as well as to perverse incentives (individual project developers having an incentive to apply for approval early in the development schedule to avoid high offset requirements), which may lead to increased short-run impacts of development and rent seeking behavior.

The Strategic Assessment strategy addresses these potential problems by anticipating the full sequence of development projects and devises an offset schedule such that equal offset obligation is assigned to each development, regardless of its position in the development schedule. This is shown by the grey dots in Figure $2 e$ (the corresponding time evolution of the system is not shown since it is close to the Sequential Compensating one). Naturally, the Strategic Assessment requires larger offsets than either the Sequential Myopic or the Sequential Correcting strategies, while the total offsets implied by the Strategic Assessment is similar to that required by developers under the Sequential Compensating strategy.

\subsection{Positive time-discounting scenario}

Offset management strategies that adopt value-to-value restoration scaling approaches, such as strategies $2-4$, raise a number of questions, including whether future utility losses and gains should be discounted. A positive discount rate $(d=5 \%)$ implies that a greater magnitude of future gains will be required to adequately compensate for the immediate losses of utility (see Figure 3, which we simplify by including only the Habitat, Utility Loss and Restoration panels) and, in our model, translates to higher requirements for 
development projects offsets. This result differs from the traditional cost-benefit analyses of conservation strategies, where higher discount rates usually entail lower conservation requirements (Stern and Great Britain. Treasury. 2007). This is because the order of ecological losses and conservation benefits is reversed in our case, with losses occurring immediately and gains being deferred due to the delays in offsetting actions and associated habitat and resource recovery processes.
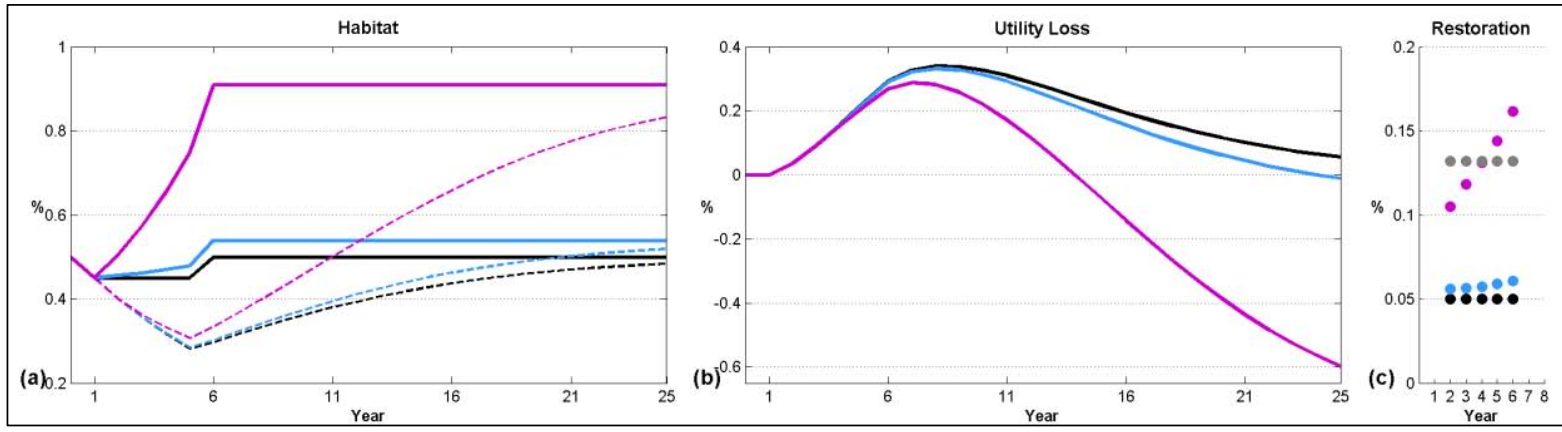

Figure 3. Reference run with a 5\% discount rate. Black denotes the Sequential Myopic, cyan the Sequential Correcting, magenta the Sequential Compensating and grey the Strategic Assessment strategy (thick lines represent the evolution of the carrying capacity / habitat carrying capacity (normalized to 1); dashed lines show the evolution of abundances). Panels (a) and (b): habitat and utility losses associated with the first three offset management strategies. Panel (c): magnitude and timing of restoration interventions required in the four offset implementation strategies.

\subsection{Initial environmental condition scenario}

Our reference simulation assumed that the environment was initially highly degraded $\left(K_{H, O}=\right.$ 0.5). As expected, a less degraded system $K_{H, O}=0.75$, allows the system to recover faster (Figure 4) regardless of the strategy adopted. While the level of offset required under the Sequential Compensating approach is now less, for the parameter values used in our simulations, implementing an offset policy in a less degraded system changes the distribution of offsets required (Figure 4c). Offsets no longer increase monotonically for developments that occur later in the sequence, but rather reach a maximum for the third development, before decreasing. Once again, a development project's position in the development schedule will affect the size of the offset which is required from this project. In this situation, assuming constant costs of offsetting effort, the incentives created for developers would be to enter early or late in the process, but avoiding seeking project approval during the middle of the development schedule. This could potentially lead to a lack of development beyond the initial projects, despite further development being considered socially worthwhile.
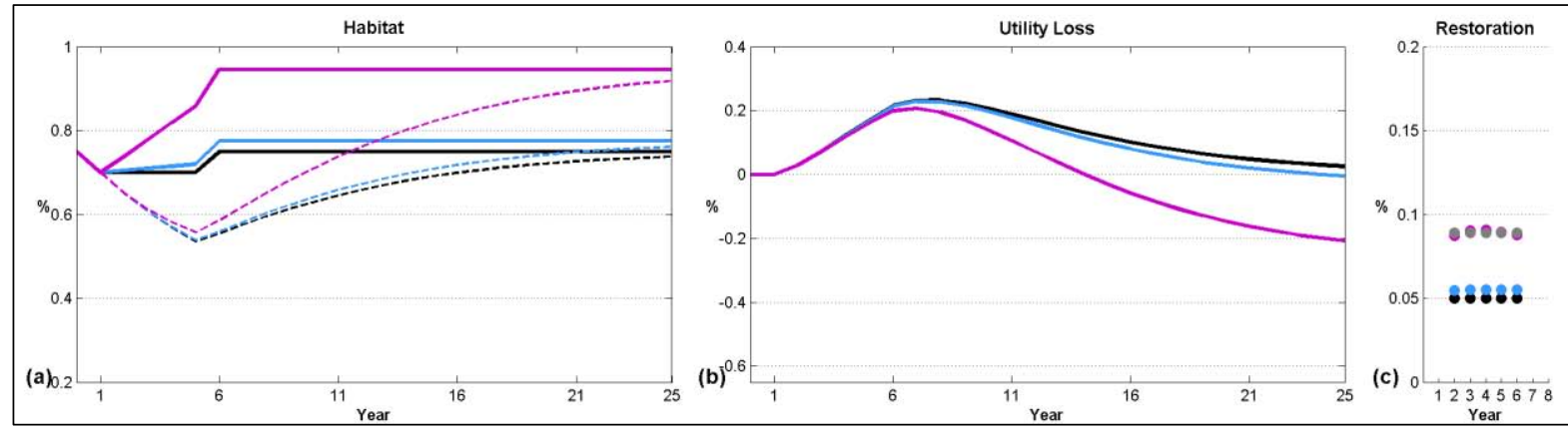

Figure 4. Reference run with an initial area available for habitat of 0.75 . Black denotes the Sequential Myopic, cyan the Sequential Correcting, magenta the Sequential Compensating 
and grey the Strategic Assessment strategy. Panels (a) and (b): habitat and utility losses associated with the first three offset management strategies (thick lines represent the evolution of the carrying capacity / habitat carrying capacity (normalized to 1); dashed lines show the evolution of abundances). Panel (c): magnitude and timing of restoration interventions required in the four offset implementation strategies.

\subsection{Social demand for offsets scenario}

The analysis so far assumes that societies threshold for action $\tau=0$ and hence an offset is imposed following the first project in the development schedule. However, for reasons discussed above, societal concern for loss of ecosystem services often arises only when cumulative loss exceeds a certain level. Below this level, the disturbances to habitat associated with individual development projects do not require offsetting. To assess the sensitivity of model outcomes to the nature of social response to ecological service loss, we analyze a scenario in which the threshold for action is $\tau=0.2$

In this case, the first offset is required in the period following the third development in the sequence, by which stage the habitat has been significantly further degraded by unrestored development occurring early in the sequence due to the cumulative impacts of the first three development projects. Offset management strategies 2 and 3, which are based on a valueto-value scaling approach, now take the current (post-development) utility level as reference $U_{\text {Ref. }}$ That is, the baseline level of utility is reset to a lower value, reflecting the reduced habitat carrying capacity and the depleted levels of resource abundance and harvest: a case of -shifting baseline" (Pauly 1995). There are several reasons why this may happen: first, the precise condition of the habitat (and hence utility) may not have been monitored prior to the first development project; second, the parties responsible for the later developments may be unwilling to assume the responsibility for compensating for the impacts of past developments; third, no legal mechanism may be in place to assign responsibility ex post to the parties responsible for the past developments. While utility losses are first experienced in time step 1 (Figure 5c), the utility losses used to calculate the level of offset required of the developers of projects 3-5 are in relation to the system state at time step 4 (Figure $5 \mathrm{~d}$ ). Not surprisingly, none of the offset management strategies explored here allow the predevelopment habitat, resource and utility levels to be recovered within the simulation period.

(a)

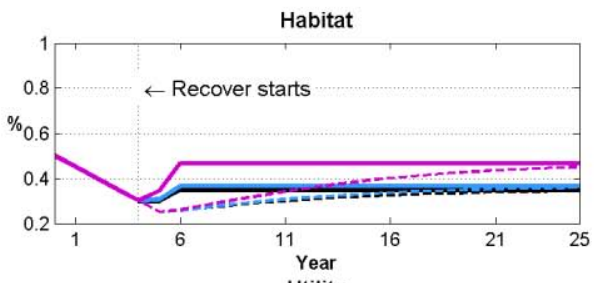

Utility

(c)
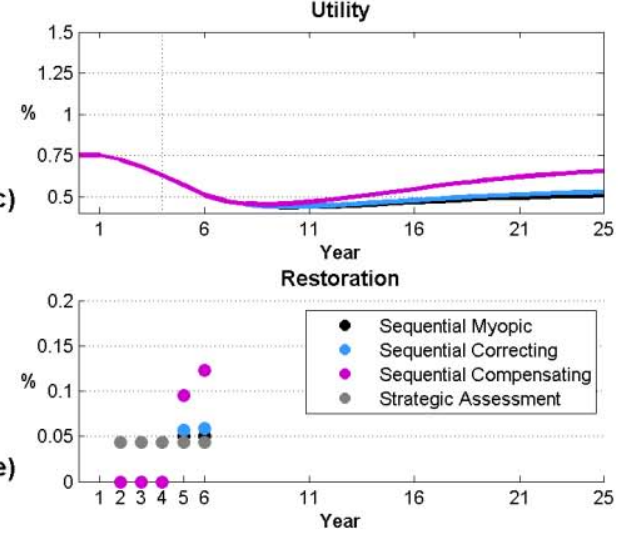

(b)

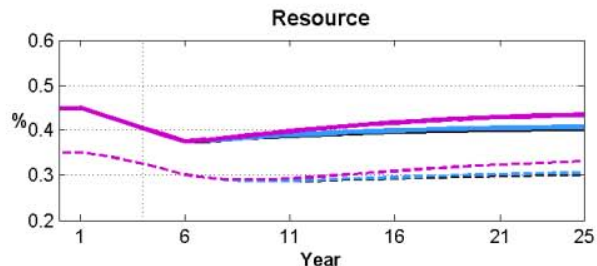

Utility Loss

(d)

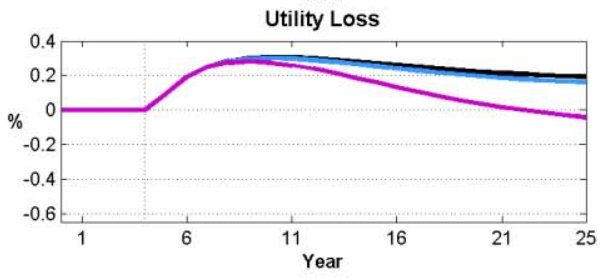


Figure 5. Reference run with positive social response threshold $\tau=0.2$. Black denotes the Sequential Myopic, cyan the Sequential Correcting, magenta the Sequential Compensating and grey the Strategic Assessment strategy. Panels (a) and (b): habitat and biological resource dynamics for the first three offset management strategies (thick lines represent the evolution of the carrying capacity $I$ habitat carrying capacity (normalized to 1); dashed lines show the evolution of abundances). Panels (c) and (d): evolution of utility and utility loss, respectively, for these three strategies. Panel (e): magnitude and timing of restoration interventions required in the four offset implementation strategies.

In Figure 6, we compare i) the impacts of time discounting, ii) the initial level of degradation of the habitat, and ii) delays in implementing offset requirements due to the nature of social responses to the loss of services, on the ability of the system to recover its pre-development level of habitat area (panel a) and on the sequence of offsetting restorations required (panel b), as discussed above.

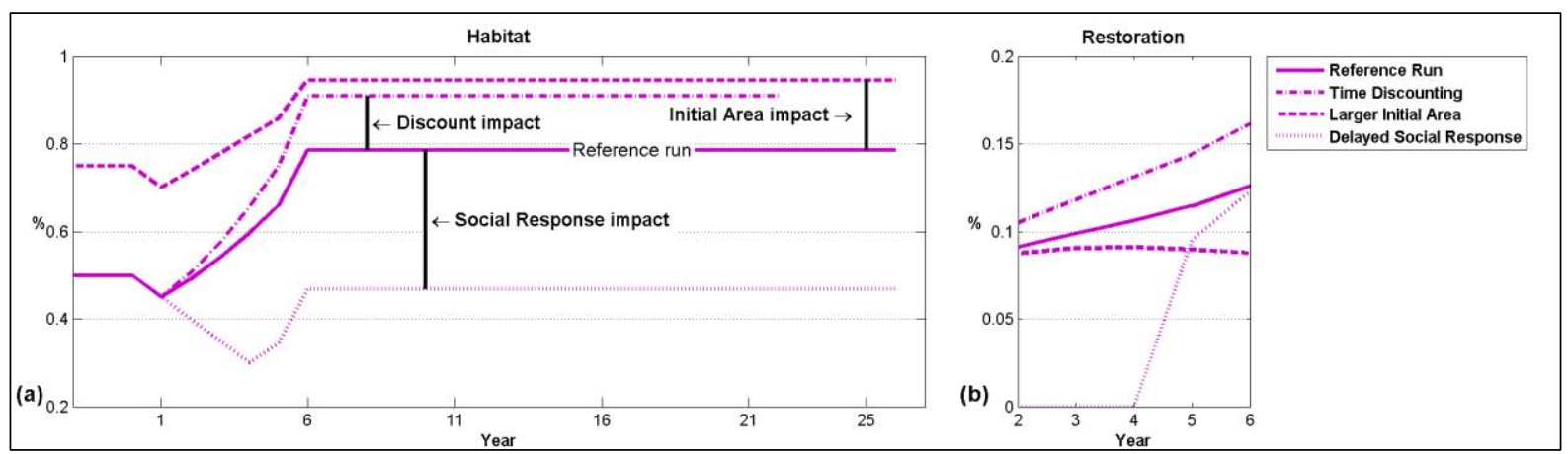

Figure 6. Comparison of the performance of the Sequential Compensating strategy in the different test cases examined. Panel (a): habitat dynamics under the different scenarios of increased discount rate, greater initial habitat area, and positive social response threshold, as compared to reference run. Panel (b): magnitude and timing of restoration interventions required under this strategy and the three different scenarios, as compared to reference run.

For the sake of conciseness, we only consider the case of the Sequential Compensating strategy. The magnitude of the impacts observed in our simulations depends on the specific parameter values defining the test cases we examine, and display a complex pattern of effects on the time schedules of required offsets (panel b) and final habitat recovery they lead to (panel a). For instance, relative to the reference run, implementing the Sequential Compensating strategy in the case of an initially less degraded habitat, will generate a proportionally smaller net gain in habitat but will reduce the offset required of each project, with projects occurring later in the development schedule now bearing proportionally less of the total responsibility for offsetting. On the other hand, accounting for a positive discount rate increases the offset requirement for all developments, particularly those that occur late in the schedule, and, relative to the reference run, results in an increase in the postrestoration level of habitat. Aggregate offset requirements are substantially reduced in the case in which there is a delay in the societal response to the loss of utility and a consequent shifting of the baseline level of utility against which losses are assessed. Furthermore, in this context, even the Sequential Compensating strategy, which accounts for cumulative impacts, does not result in full recovery of the habitat to its pre-development level. 


\section{Discussion and conclusion}

Our primary objective in this paper was to explore several conceptual issues relating to the effectiveness of alternative biodiversity offsetting approaches, using a marine system as an example. We did this by developing a stylized modeling framework to assess offset management strategies under alternative scenarios relating to ecological and societal responses to the implementation of multiple developments and offset actions. The results of our simulations of a reference run (Figure 2) are reproduced in Table 1 highlighting the size of offsets and utility losses associated with the four offset strategies explored. Here the individual project and cumulative offset magnitudes are expressed as a multiple of $D$, the magnitude of the loss of available habitat arising from a single development project, and utility losses are integrated over the entire time horizon.

Table 1. Comparison of the aggregate utility loss over the entire projection horizon for the reference run

\begin{tabular}{|c|c|c|c|c|}
\hline Strategy & $\begin{array}{c}\text { Offset requirement } \\
\text { schedule } \\
C_{t=1 \rightarrow 5}\end{array}$ & $\begin{array}{c}\text { Total offset } \\
\text { requirement } \\
\sum C_{t=1 \rightarrow 5}\end{array}$ & $\begin{array}{c}\text { Utility } \\
\text { Loss } \\
\text { final } \\
\text { period } \\
L_{U, t=20}\end{array}$ & $\begin{array}{c}\text { Utility Loss } \\
\text { time } \\
\text { horizon } \\
\sum L_{U, t=1 \rightarrow 20}\end{array}$ \\
\hline $\begin{array}{l}\text { Sequential } \\
\text { myopic }\end{array}$ & $(1.00,1.00,1.00,1.00,1.00)$ & 5 & 0.06 & 4.60 \\
\hline $\begin{array}{l}\text { Sequential } \\
\text { correcting }\end{array}$ & $(1.12,1.13,1.15,1.18,1.22)$ & 5.80 & 0 & 3.89 \\
\hline $\begin{array}{l}\text { Sequential } \\
\text { compensating }\end{array}$ & $(1.82,1.97,2.12,2.29,2.52)$ & 10.72 & -0.41 & -0.12 \\
\hline $\begin{array}{l}\text { Strategic } \\
\text { assessment }\end{array}$ & $(2.10,2.10,2.10,2.10,2.10)$ & 10.50 & -0.40 & -0.11 \\
\hline
\end{tabular}

The Sequential Myopic strategy involves the smallest offset effort but results in a decline in resource abundance, and hence fails to achieve the no-net utility loss objective defined with respect to either $L_{U, t=20}$ or $\sum L_{U, t=1 \rightarrow 20}$. The Sequential Correcting strategy guarantees no netutility loss in terms of returning the value of the services provided by the resource to its predevelopment level by the end of the simulation period. Nevertheless, despite the increase in the area of available habitat, this strategy results in a net loss of cumulative utility. The Sequential Compensating approach has as its goal no net loss in cumulative utility and in our simulations even provides for utility gains towards the end of the time period. However, this involves re-creating carrying capacity for the habitat to a level which is significantly higher than the baseline level, and increasingly so with higher discount rates. Consequently, in the absence of any further developments, the resource will continue to provide a flow of utility that exceeds its pre-development level, beyond the 20-year period horizon. The same conclusion applies to the Strategic Assessment. An interesting question thus arises as to who should be entitled to receive such surplus - i.e. does it create credits for the developer against which future development may be traded or does it generate a windfall gain for society?

The offset management strategies explored in this paper also imply different total levels of offset and distributions of these efforts (and hence costs) across projects, depending on their temporal position in the development schedule. For our reference simulations, for example, total required offset is more than twice as high for the Strategic Assessment and 
Sequential Compensating strategies than for the Sequential Myopic strategy. In addition, not only may the Sequential Compensating approach lead to situations in which the requested offsets change as a function of the intervention time, but also these changes may not be monotonic, potentially leading to complex economic (dis)incentives. Further understanding the way in which alternative offset management strategies impact expected offset obligations, and their effect on developer behavior and hence on the level and timing of development will be important.

Thus, a key result of this analysis is that, if the no net loss objective is to be taken seriously, cumulative impacts would need to be considered strategically, as this would seem to both enable the objective to be achieved, and align the incentives for developers across development projects in a given area. In addition, in evaluating the need for stringent offsetting policies, our model predicts that if offsets are managed using a Sequential Myopic strategy, developers on whom the offset burden falls will have an incentive to seek out relatively less damaged ecosystems with a high social threshold, for their development projects. Hence there may be interactions between the ways in which biodiversity offset policies are implemented in different areas or jurisdictions that also need to be considered in developing these policies.

A related dimension which would require further investigation is the potential consequence of some of the uncertainties highlighted above for offset banking. As stressed by Vatn (2014), banking may play a key role in implementing biodiversity offsets in practice, by allowing the pooling of offsets in order to improve the efficiency of restoration actions. Banking can also be used to provide inter-temporal flexibility in the development and use of offsets, as has been implemented in a wide range of tradeable permit programs for environmental management (Call and Lew 2015). However, such pooling arrangements will strongly depend on the capacity of the regulator to establish and follow the evolution of requirements regarding habitat restoration and the recovery of ecosystem services, across the different sites included in an offsetting strategy. This has for example been shown to play an important role in wetland mitigation banking in the United States (Vaissière and Levrel 2015). The simulations also show that in an ecosystem initially less severely degraded, the total offsetting effort required for a given development schedule may be less than when the system is more degraded. Where this is observed, there may be increasing marginal offset costs of development associated with the degradation of ecosystems, and a case for early offset action in the process of developing relatively undisturbed habitats, as such development increases the opportunity cost of foregone habitat conservation. Additional issues that could be investigated here relate to the implications of potential correlations of the social response thresholds with the initial condition of the ecosystem, or with discount rates.

While the analysis presented here focuses on a habitat-dependent fisheries production issue, our modeling framework can readily be adapted to account for either more or less complicated ecosystem structures and for cases in which the value of ecosystem services may not be strictly related to extractive resource harvesting (Engle 2011, Stoeckl et al. 2011). Depending on the context, the perception of losses in ecosystem services may be different, with society perhaps being more willing to accept some loss in a commercial fishery than in the population of an endangered species. The inclusion of a social response function in the model provides a means to explore the implications of such differences for offset management strategies. From a broader biodiversity conservation perspective, a key challenge in further exploring such trade-offs however remains to identify those indices that could reflect changes at multiple levels of diversity in an ecosystem, as well as the consequences of these changes on ecosystem services and their social perception.

Our analysis has let us explore several important issues related to the use of offsets as a biodiversity conservation instrument, within a modeling framework that has captured elements of the complexity characterizing marine systems. Nevertheless, there are a 
number of important issues that our analysis does not address. For instance, the offset management strategies examined do not allow for pre-emptive offsets, that is where policy requires offsets to be implemented prior to development. While this has the potential to avoid the loss in utility arising from the implementation of the development schedule, the use of anticipatory approaches presents other difficulties. These include uncertainty as to the actual habitat damage incurred during the development (so no net loss is not guaranteed if offsets are approved ex ante), as well as regulatory risk to the developer (in that changes in offset policy, offset management strategy or societal concern between offset establishment and full functionality, may jeopardize project approval). This will be particularly problematic under the Sequential Correcting strategy where other unanticipated developments may start earlier and the anticipatory offset may no longer be sufficient. Hence the pre-emptive offset approach may entail dis-incentives for development unless a myopic offset policy is employed, which leaves society exposed to the possibility of declining habitat, resource and associated utility.

In contrast, ex post offsets may allow for greater flexibility in terms of offset requirements, so that actual rather than anticipated impacts can be properly compensated. However, although not explored in this paper, the risk of an offset action not producing the anticipated outcome in terms of biodiversity or habitat recovery due to implementation, technical or governance failure, challenges the ability of offset policies based on ex post implementation of offsets to deliver no net loss, and hence the promised reconciliation of economic development with conservation objectives. These various sources of uncertainty may themselves impact the functioning of offset banking strategies where these can be implemented, driving expectations as to possible changes in the value of current and future bankable offsets, and leading to complex investment strategies with consequences on the effectiveness of offsets policies that would need to be further explored.

Results from this stylized model highlight some of the key empirical issues which need to be further explored in developing offset policy, particularly in environments affected by the cumulative impacts of sequential developments requiring -sets of offsets". In particular, it is apparent that the distribution of offset responsibility across developers will depend on the offset strategy and empirical context in which it is applied. In addition, the incentives may be affected by the nature of two key responses to offsets, relating to: (i) the ecological dynamics underlying recovery of the ecosystem; and (ii) societal responses to changes in services and the associated utility. Increased empirical understanding of these responses would provide knowledge that would be useful in evaluating preferred management strategies for biodiversity offsets. Finally, in the context explored here, discounting of future benefits and costs implies higher requirements for offsets, while the impact of discounting on the development-versus-biodiversity-conservation trade-off is usually cast such that higher discount rates lead to lower conservation incentives (Clark and Lamberson 1982). This result is easily explained by the fact that achieving a given level of discounted utility with offsets that provide delayed benefits requires greater immediate conservation efforts.

\section{Acknowledgments}

This work was supported by the Marine Biodiversity Hub of the Australian Government's National Environmental Research Program (NERP), under Theme 2 on Supporting Management of Marine Biodiversity", and by CSIRO's Wealth from Oceans Flagship and Oceans and Atmosphere Flagship, as well as by Ifremer's Biological Resources \& Environment Department. 
Anderson, E. E. 1989. Economic Benefits of Habitat Restoration: Seagrass and the Virginia Hard-Shell Blue Crab Fishery. North American Journal of Fisheries Management 9:140-149.

Barbier, E., I. Strand, and S. Sathirathai. 2002. Do Open Access Conditions Affect the Valuation of an Externality? Estimating the Welfare Effects of Mangrove-Fishery Linkages in Thailand. Environmental and Resource Economics 21:343-365.

Boncoeur, J., F. Alban, O. G. Ifremer, and O. T. Ifremer. 2002. Fish, fishers, seals and tourists: Economic consequences of creating a marine reserve in a multi-species, multi-activity context. Natural Resource Modeling 15:387-411.

Call, I. L. and D. K. Lew. 2015. Tradable permit programs: What are the lessons for the new Alaska halibut catch sharing plan? Marine Policy 52:125-137.

Clark, C. W. and R. Lamberson. 1982. An economic history and analysis of pelagic whaling. Marine Policy 6:103-120.

Department of Sustainability, E., Water, Population and Communities. 2012. Environment Protection and Biodiversity Conservation Act 1999 Environmental Offsets Policy. Page 30 in E. Department of Sustainability, Water, Population and Communities, editor., Canberra.

Engle, V. 2011. Estimating the Provision of Ecosystem Services by Gulf of Mexico Coastal Wetlands. Wetlands 31:179-193.

Foley, N. S., C. W. Armstrong, V. Kahui, E. Mikkelsen, and S. Reithe. 2012. A Review of Bioeconomic Modelling of Habitat-Fisheries Interactions. International Journal of Ecology 2012.

Foley, N. S., V. Kahui, C. W. Armstrong, and T. M. Van Rensburg. 2010. Estimating Linkages between Redfish and Cold Water Coral on the Norwegian Coast. Marine Resource Economics 25:105-120.

Gardner, T. A., A. Von Hase, S. Brownlie, J. M. M. Ekstrom, J. D. Pilgrim, C. E. Savy, R. T. T. Stephens, J. O. Treweek, G. T. Ussher, G. Ward, and K. Ten Kate. 2013. Biodiversity Offsets and the Challenge of Achieving No Net Loss. Conservation Biology:n/a-n/a.

Gordon, A., W. T. Langford, J. A. Todd, M. D. White, D. W. Mullerworth, and S. A. Bekessy. 2011. Assessing the impacts of biodiversity offset policies. Environmental Modelling \& Software 26:1481-1488.

Knowler, D. J., B. W. MacGregor, M. J. Bradford, and R. M. Peterman. 2003. Valuing freshwater salmon habitat on the west coast of Canada. Journal of Environmental Management 69:261-273.

Lindholm, J. B., P. J. Auster, M. Ruth, and L. Kaufman. 2001. Modeling the Effects of Fishing and Implications for the Design of Marine Protected Areas: Juvenile Fish Responses to Variations in Seafloor Habitat. Conservation Biology 15 (2): 424-437.

Modelado de los Efectos de la Pesca y sus Implicaciones para el Diseño de Areas Marinas Protegidas: Respuesta de Peces Juveniles a Variaciones en el Hábitat del Fondo Marino. Conservation Biology 15:424-437.

Lynne, G. D., P. Conroy, and F. J. Prochaska. 1981. Economic valuation of marsh areas for marine production processes. Journal of Environmental Economics and Management 8:175-186.

Madsen, B., N. Carroll, D. Kandy, and G. Bennett. 2011. State of Biodiversity Markets. Forest Trends, Washington, DC.

Maron, M., R. J. Hobbs, A. Moilanen, J. W. Matthews, K. Christie, T. A. Gardner, D. A. Keith, D. B. Lindenmayer, and C. A. McAlpine. 2012. Faustian bargains? Restoration realities in the context of biodiversity offset policies. Biological Conservation 155:141148.

Millennium Ecosystem Assessment. 2005. Ecosystems and Human Well-being: Synthesis. Island Press, Washington, DC. 
Mitchell, R. E. and J. R. Parkins. 2011. The challenge of developing social indicators for cumulative effects assessment and land use planning. Ecology and Society 16:29.

Moilanen, A., A. J. A. Van Teeffelen, Y. Ben-Haim, and S. Ferrier. 2009. How Much Compensation is Enough? A Framework for Incorporating Uncertainty and Time Discounting When Calculating Offset Ratios for Impacted Habitat. Restoration Ecology 17:470-478.

Morris, R. K. A., I. Alonso, R. G. Jefferson, and K. J. Kirby. 2006. The creation of compensatory habitat-Can it secure sustainable development? Journal for Nature Conservation 14:106-116.

Overton, J., R. T. T. Stephens, and S. Ferrier. 2013. Net Present Biodiversity Value and the Design of Biodiversity Offsets. Ambio 42:100-110.

Palmer, M. A. and S. Filoso. 2009. Restoration of Ecosystem Services for Environmental Markets. Science 325:575-576.

Pauly, D. 1995. Anecdotes and the shifting baseline syndrome of fisheries. Trends in Ecology \& Evolution 10:430.

Pickett, E., M. Stockwell, D. Bower, J. Garnham, C. Pollard, J. Clulow, and M. Mahony. 2013. Achieving no net loss in habitat offset of a threatened frog required high offset ratio and intensive monitoring. Biological Conservation 157:156-162.

Stern, N. H. and Great Britain. Treasury. 2007. The economics of climate change : the Stern review. Cambridge University Press, Cambridge, UK ; New York.

Stoeckl, N., C. C. Hicks, M. Mills, K. Fabricius, M. Esparon, F. Kroon, K. Kaur, and R. Costanza. 2011. The economic value of ecosystem services in the Great Barrier Reef: our state of knowledge. Annals of the New York Academy of Sciences 1219:113-133.

Vaissière, A.-C. and H. Levrel. 2015. Biodiversity offset markets: What are they really? An empirical approach to wetland mitigation banking. Ecological Economics 110:81-88.

Vatn, A. 2014. Markets in environmental governance. From theory to practice. Ecological Economics. 


\section{Details of the bio-economic model}

The biophysical component of the model is adapted from the formal representation of habitat - species interactions proposed by (Foley et al. 2012). Specifically, we consider a biological resource the biomass of which grows according to:

$$
F_{t}\left(X_{t}, H_{t}\right)=r_{X}\left(H_{t}\right) X_{t}\left(1-\frac{X_{t}}{K_{X_{t}}\left(H_{t}\right)}\right)
$$

Where $\mathrm{X}$ is the biomass of the biological resource, $H$ represents the habitat and $t$ denotes time. The habitat impacts the biological resource through its effect on both the resources' intrinsic ability to grow $r_{X}()$ and its carrying capacity $K_{X}()$.

The biological resource provides a number of ecosystem services, which may include provisioning services (e.g. commercial or subsistence fishing, extraction of molecules with medicinal properties), cultural services (e.g. recreational fishing or diving, aesthetic services), as well as supporting and regulating services (Millennium Ecosystem Assessment 2005).

We limit the scope of our analysis to consider the case of an extractive use, such as commercial fishing, where:

$$
h\left(X_{t}\right)=q E X_{t}
$$

where $\mathrm{h}$ is harvest, $E$, considered to be fixed, is the effort devoted to extracting or harvesting the biological resource, and $q$ is a technical parameter representing the proportion of the resource removed by a unit of harvesting effort. Effort is also assumed to be such that, given the ecological system under consideration, the resource cannot be driven to extinction if sufficient habitat is maintained.

Our focus on a single, extractive service or output does not limit the generality of our model. While posing various valuation challenges, the formulation proposed can easily be adapted to consider the case of non-extractive uses of the ecosystem, such as recreational diving or eco-tourism, and non-use values including those for cultural and regulating services. Such an activity could for example be represented as in (Boncoeur et al. 2002), by a marine ecotourism function $Y\left(X_{t}\right)=a X_{t}^{b} E^{c}$ where $Y$ is the number of visits, $E$ represents the production effort devoted to the promotion of ecotourism in the area considered, $a$ is $a$ positive dimension parameter, and $b$ and $c$ are the elasticity of visits with regards to the abundance of the biological resource and to production effort respectively.

We assume a market exists for the harvest, such that a utility function $U_{t}$ can be defined as:

$$
U_{t}=p h\left(X_{t}\right)-c
$$

with $p$ and $c$ being fixed parameters representing the price of a unit of the harvested resource and the unit cost of effort respectively. With $E$ fixed, $U_{t}$ is a linear function of $X_{t}$. In discrete time, the biological resource is assumed to evolve according to:

$$
X_{t+1}=X_{t}+F\left(X_{t}, H_{t}\right)-h\left(X_{t}\right)
$$

Following (Foley et al. 2012), we assume that the habitat, $H$, influences the growth of the biological resource as follows: 


$$
\left[\begin{array}{l}
r(H)=a+b H \\
K(H)=f+g H
\end{array} \quad a, b, f, g \geq 0\right.
$$

Where parameters $a, b, f$ and $g$ depend on the nature of the relationship between the habitat and the productivity and carrying capacity of the biological resource. As noted by (Foley et al. 2012), $a=0$ and $f=0$ ( $v s$. $a>0$ and $f>0$ ) correspond to cases where the habitat is essential (vs. facultative) to the sustainability of the biological resource. In reality, the relations between habitat and the intrinsic growth and carrying capacity of the biological resource are likely to display saturation. It is assumed here that the linearity of these relations is valid only over an intermediate domain of habitat size. The existence of the habitat itself is assumed to depend on a number of environmental conditions (for example, the habitat may depend on inflow of freshwater of a certain quality), which determine a carrying capacity for the type of habitat considered, $K_{H}$. This suggests how habitat restoration under an offset might be given effect in practice.

We distinguish between disturbances and recovery processes of the habitat and disturbances and recovery processes of the area available for habitat. Disturbances to the habitat can occur naturally (e.g. through storm damage), and hence we model habitat recovery, again assuming a logistic growth function, as:

$$
\begin{aligned}
& H_{t+1}=H_{t}+G\left(H_{t}\right) \\
& G\left(H_{t}\right)=r_{H} H_{t}\left(1-\frac{H_{t}}{K_{H}}\right)
\end{aligned}
$$

With $r_{H}$ the intrinsic ability for the habitat to recover, and $\mathrm{K}_{H}$ the maximum area available for the habitat (i.e. the natural" level of the habitat in an undisturbed situation), hereafter called the habitat carrying capacity".

In contrast, disturbances to the area available for habitat occur as a result of development projects, which also remove a corresponding fraction of the habitat. We define $D_{t}$ as the amount of habitat and habitat carrying capacity that is disturbed (removed) by each project at the beginning of a given time step:

$$
\widetilde{H_{t}}=H_{t}-D_{t} ; \widetilde{K}_{H}=K_{H}-D_{t}
$$

Where $\widetilde{H_{t}}$ is the effective amount of post-development habitat and $\widetilde{K}_{H}$ is the revised habitat carrying capacity. Importantly, while habitat recovery occurs naturally according to equations (s6) and (s7), recovery of the habitat carrying capacity can only occur following a compensating restoration, $\mathrm{R}$, implemented as a result of the biodiversity offset policy, as described in the article.

\section{The Social response function}

The social response function represents the relation between an actual utility loss and the level of social concern it generates, and can take different forms. To allow for a wide range of scenarios, we use the Fermi function and define $s$ as follows:

$$
S=\frac{1}{e^{\left(\frac{L_{0}-L_{U}}{L_{S}}\right)}+1}
$$

$L_{0}$ determines the existence and location of an inflexion point in the social response function. This is where the gradient of the response function is steepest, that is where the social concern is most sensitive to utility loss. $L_{S}$ determines the steepness of the social response function around the threshold for action and, in combination with $L_{0}$, the range of utility loss over which the social response is effectively impacted. An example is given in Figure 6 
which illustrates two sets of social response functions. The $\mathrm{x}$-axis represents utility loss and $y$-axis the social concern (s). Two sets of social response curves are shown, characterised by $L_{0}=0.2$ (left) and $L_{0}=0.5$ (right), respectively. For each set, five curves are shown, with slopes $L_{s}$ ranging from 0.05 to 0.2 . Depending on the $L_{s}$ value, the social concern increases more or less abruptly when the utility loss approaches $L_{0}$. When the social concern exceeds the Threshold for Action' (here arbitrarily set to 0.2) the management body has to respond by imposing some form of restoration initiatives. As show in Figure s6, this threshold can be reached for very different values of utility loss (from less than $10 \%$ to more than $40 \%$ in this example), depending on the specific values of $L_{0}$ and $L_{s}$. Further exploration of the function's behaviour is possible, but would be best informed by empirical understanding of real life case studies.

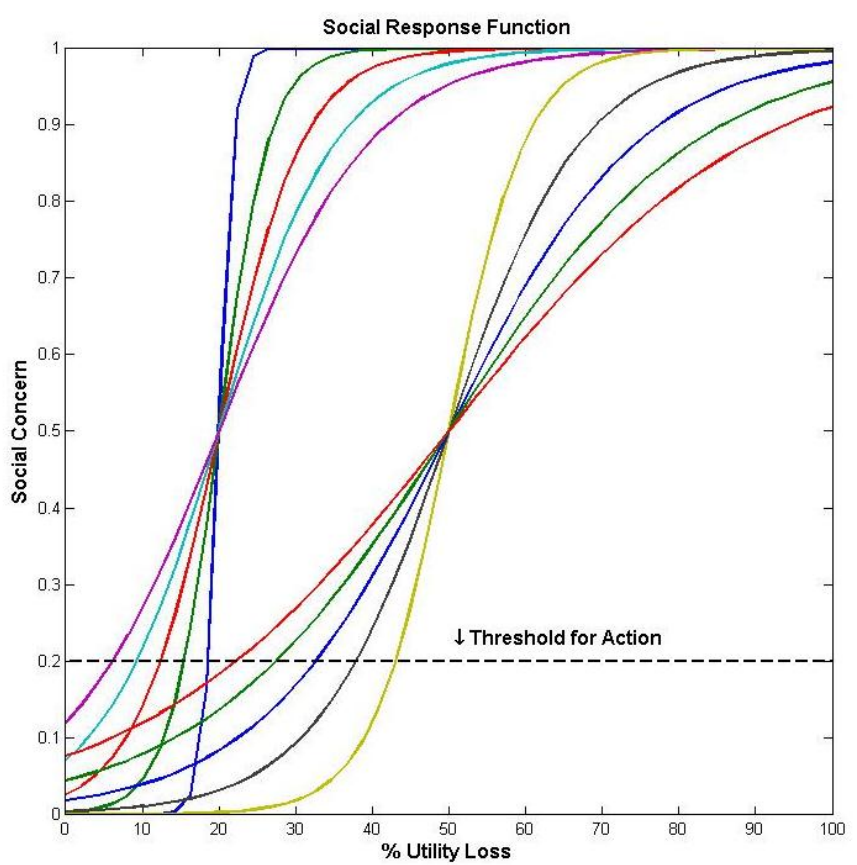

Figure 7. Two sets of possible social response functions. $X$-axis: Actual Utility Loss as a \% of utility without development. Y-axis: Social Concern factor. Curves of different colours represent different values of Fermi function parameters

\section{Model parameterization}

The model has been implemented in Matlab. The initial values of state variables and model parameters used are presented in tables 2 and 3 respectively. For the values which change according to the simulation, we include the ranges used.

Table 2 - Initial conditions used in simulations

\begin{tabular}{clc}
\hline Variable name & Definition & Value \\
\hline $\boldsymbol{K}_{H, 0}$ & Initial area available for the supporting habitat & $0.5-0.75$ \\
$\boldsymbol{H}$ & Habitat size & $\boldsymbol{K}_{H, 0}$ \\
$\boldsymbol{h}_{0}$ & Ecosystem service (harvest of the biological resource) & 1 \\
$\boldsymbol{D}$ & Loss of $K_{H}$ from a single development project & 0.05 \\
$\boldsymbol{E}$ & Effort devoted to extracting or harvesting the biological resource & 1 \\
\hline
\end{tabular}


Table 3 - Parameter values used in simulations

\begin{tabular}{clr}
\hline Parameter & Definition & Value \\
\hline $\mathbf{q}$ & Catchability coefficient for biological resource & 0.1 \\
$\mathbf{a}$ & Constant in biological resource growth function & 0.3 \\
$\mathbf{b}$ & Habitat dependent factor in biological resource growth & 0.3 \\
$\mathbf{f}$ & Constant in biological resource carrying capacity function & 0.3 \\
$\mathbf{g}$ & Habitat dependent factor in biological resource carrying & 0.3 \\
$\boldsymbol{r}_{\boldsymbol{H}}$ & $\boldsymbol{H}$ growth & $0.15-0.25$ \\
$\boldsymbol{K}_{\text {Hmax }}$ & Normalised H maximum carrying capacity & 1 \\
$\mathbf{p}$ & Unit price of ecosystem service (harvest) & 50 \\
$\mathbf{c}$ & Unit cost of harvest & 1 \\
$\mathbf{d}$ & Discount rate & $0-0.05$ \\
$\boldsymbol{\alpha}$ & Maximum social response multiplier & 1.5 \\
$\boldsymbol{\tau}$ & Threshold for action & $0-0.2$ \\
$\boldsymbol{L}_{\boldsymbol{0}}$ & Social response threshold inflexion point coefficient & 0.20 \\
$\boldsymbol{L}_{\boldsymbol{S}}$ & Social response steepness coefficient & 0.05 \\
$\mathbf{z}$ & Amount of restored $K_{H}$ per unit of offsetting effort & $\mathrm{D}$ \\
\hline
\end{tabular}

These parameter values were chosen based on a partial analysis of model sensitivity (not included) around the values of parameters $a, b, f, g, r_{H}, d$. The values correspond to $a$ resource that moderately depends on the habitat for both its growth and carrying capacity and for which the greatest difference between offset strategies in terms of total restoration effort required occurs. The partial sensitivity analysis also showed that the habitat growth rate and discount rate only have second order effects. 